\title{
DYNAMICS AND CONTROL OF LOOP REACTORS: A REVIEW
}

\author{
Moshe Sheintuch* And Olga Nekhamkina
}

\begin{abstract}
In the loop reactor (LR) the system is composed of several reactor units that are organized in a loop and the feeding takes place at one of several ports with switching of the feed port in a periodic way. In its simplest operation a pulse is formed and rotates around it, producing high temperatures which enable combustion of dilute streams. A limiting model with infinite number of units was derived. Rotating pulses, that are steady in a coordinate moving with the switch velocity, emerge in both asymptotic and discrete models when the ratio of switching to front propagation velocities is around unity. But this behavior exists over a narrow domain of this ratio. Simulations were conducted with generic first order Arrhenius kinetics. Experimental observations of simple frozen rotating pulses are reviewed. Outside the narrow frozen rotating patterns domain the system may exhibit multi- or quasi-periodic operation separated by domains of inactive reaction. The bifurcation set incorporates many 'finger'-like domains of complex frequency-locked solutions that allow to significantly extend the operation domain with higher feed temperature or concentration. Control is necessary to attain stable simple rotating frozen patterns within the narrow domains of active operation. Various control approaches that were suggested, or experimentally applied for this purpose, are reviewed. Actual implementation of combustion in LR will involve several reactants of different ignition temperatures and varying concentration. Design and control should be aimed at producing locked fronts and avoid extinction of the slower reaction.
\end{abstract}

Mathematics Subject Classification. 35A18, 35B35, 35B36, 35B40, 35K57, 37G99, 93C20.

Received October 21, 2020. Accepted June 10, 2021.

\section{INTRODUCTION}

Most of the processes in the chemical and petrochemical industries are catalytic, usually in packed beds in which the catalyst phase is solid and stationary. The packed bed, which is the 'working horse' of the industry, is just a large tube packed with a catalyst, where the reactant stream enters at one port and leaves at the opposite port (once-through operation). If the system is adiabatic and at steady state and the reaction is exothermic, the maximal temperature rise is limited by the reactant concentration (see the exact expression below) and this is the highest temperature in the system. This temperature, however, may be too small for appreciable conversion. This is especially so in environmental abatement applications, where diluted stream of (sometimes toxic) reactants (e.g., methane, hydrocarbons, $\mathrm{CO}$, ammonia etc.) are burned into harmless $\mathrm{CO}_{2}, \mathrm{~N}_{2}$ and water instead of dispersion in the atmosphere. However, the temperature of a front $\left(T_{m}\right)$ moving downstream at a

Keywords and phrases: Moving pulses, network of chemical reactors, loop-reactor, distributed systems, control, control of distributed system, bifurcations.

Department of Chemical Engineering, Technion - I.I.T., Technion City, Haifa 32 000, Israel.

* Corresponding author: cermsll@technion.ac.il 

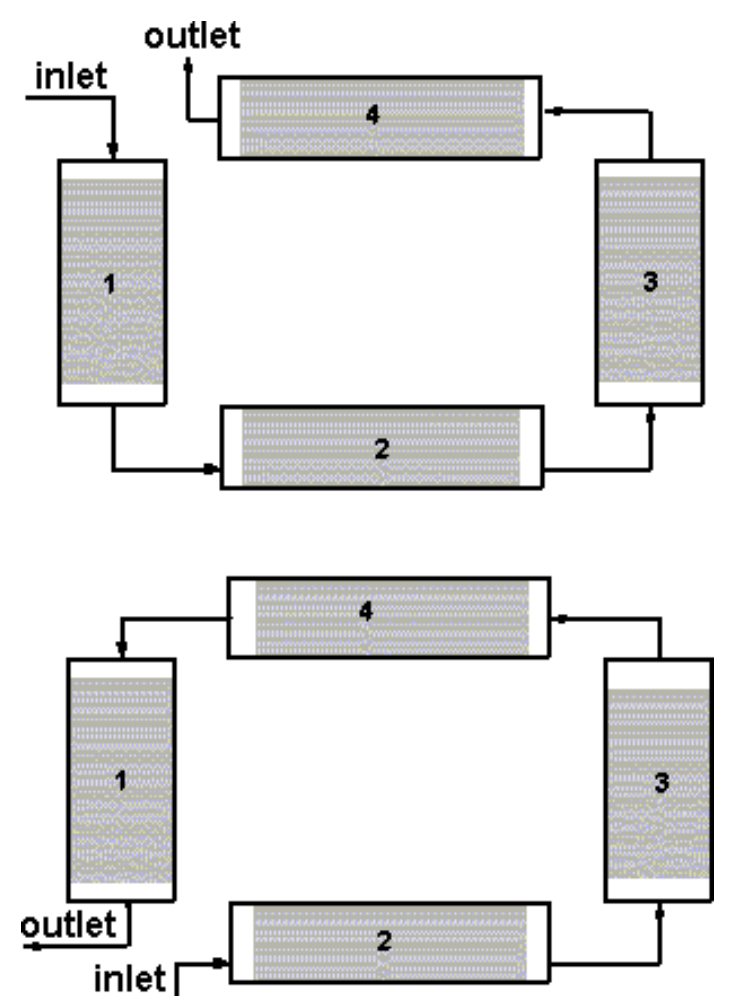

Figure 1. Two consecutive feed positions (out of 4) in a four-unit loop-reactor.

velocity $V_{f r}$ in a once-through system can reach a value that significantly exceeds the adiabatic temperature $\left(T_{a}\right)$. The ratio of the maximal to the adiabatic temperature rise for an adiabatic system is

$$
\frac{\Delta T_{m}}{\Delta T_{a}}=\frac{T_{m}-T_{i n l}}{T_{a}-T_{i n l}}=\frac{u-V_{f r}}{u-L e V_{f r}}
$$

In real systems, the ratio of the solid to the fluid capacities obeys $L e \gg 1$ and $V_{f r} \ll u$ (the convective velocity, see below) and high temperatures can be sustained as long as the front stays within the system. Thus, for that purpose some kind of periodic boundary conditions must apply.

In the loop reactor (LR), which is also referred to as a network (NTW) reactor, a process which has been a subject of research by several groups, for the past 20 years or so, the reactor is organized as a loop and a pulse is formed and rotates around it. In this reactor the feeding takes place at one of several or many ports. To illustrate this concept consider a loop reactor with $N$ units $(N=2,3, \ldots)$ with a feed-port and an exit-port that are switched every predetermined time interval. All $N$ units are employed at all times: In the first interval we feed through unit 1 while the flow exits at the end of unit $N$; in the second interval the flow enters at unit 2 and exits from unit 1, and so on (see Fig. 1 for two steps in a 4-reactor system). Thus, the feed and exit streams are adjacent, which requires special arrangement and control of valves. The idea was suggested in reference [27], the simulations were conducted for a case of two units [16] and extended for a three-unit system in the following works $[6,7,17,26]$. Several studies considered a LR (or a NTW) with the feed port jumping several units in every shift $[3,24,25]$. Such a strategy is claimed to allow better control, since it allows to increase the switching time, as we describe below, the main properties of the LR behavior are captured with a strategy using a one-unit shift. 
Other solutions for attaining temperatures that exceed the adiabatic rise were also suggested: In the reverse flow reactor (RFR [28]), that is also based on operating under forced periodic flow conditions, the flow direction is alternated periodically (every 5-10 minutes): the temperature profile is symmetric and two fronts (on the left and right side) are trapped while their positions oscillate in time. The advantage of such reactors over simple once-through operation for attaining high temperatures has been demonstrated in many studies of adiabatic units (e.g., see [11, 19], for review). In yet another approach [20] the reactor is equipped with a heat exchanger that use the exit stream to ignite the feed.

The loop reactor(s) described above are two of a small set of processes that employ loop-shaped units for reaction or separation of a continuous process and we believe that this class, that employ periodic boundaryconditions for some of the variables, may yield novel important applications. Another member in this group is the 'simulated moving bed reactor' that are used for continuous chromatographic separation of materials [8].

The aim of this article is to review the mathematical nature of dynamics and control of loop reactors, which may be of importance in designing such a system: We focus on a $T$-periodic solution with a one-unit switching strategy; this strategy admits an asymptotic solution. $(N \rightarrow \infty$, the switching time $\sigma \rightarrow 0$, but a finite switching velocity). Such limiting solutions are reviewed in Section 2 , with slow or intermediate switching velocities, and we compare the dynamics of asymptotic and discrete (finite N) models. Results show that high-temperatures moving $T$-patterns can be sustained in a narrow domain of parameters, but other wider domains exist characterized with complex behavior. The switching strategy with several unit jump is considered in Section 2.4. We also review some experimental results in Section 2.3 and their relevance to the theory. Keeping such a state in a system with uncertainties of parameters, will require a control scheme, as reviewed in Section 3. In Section 4 we expand the application of a loop reactor to combustion of a mixture of reactants, where several fronts with essentially different temperatures and concentrations may form. We conclude by pointing out open problems that we deem to be of importance in bringing this technology to maturity.

\section{Asymptotic solution}

The authors [37] studied a discrete system and the asymptotic solutions of loop reactors, in which a single exothermic reaction occurs. Since the residence time in the reactor is typically $1 \mathrm{~s}$ or a fraction of it, while the front velocity is typically of $O(0.001 \mathrm{~m} / \mathrm{s})$, then a noticeable front displacement will take, therefore, 1000 $\mathrm{s}$ or so. Between these two time scales, we can switch a port every 10-200 s. After switching it will take few seconds (i.e., several residence times) for the mass-balance to readjust itself, while the temperature profile can be assumed to be constant for that duration. The difference of the time scales is an essential prerequisite for emergence of various patterns which can be controlled in a loop reactor by appropriate choice of the switching intervals (external forcing).

We start by reviewing the mathematical model of a discrete system and analysis of its properties. These will be shown to converge to an asymptotic model (with infinite $N$ ), and the two are compared at each step. The analysis considered a pseudo-homogeneous (no gradients between phases) one-dimensional model of an adiabatic catalytic bed reactor in which one or several 1st order Arrhenius reactions occur $\left(i=1,2, \ldots, N_{s}\right.$ is the component number, $k=1,2, \ldots, N_{r}$ is the reaction number). In most cases we employ one reaction only but the model below accounts for the general case. Consider a loop reactor of $N$ identical units (each one of the length $\Delta L=L / N$, Fig. 1) with gradual switching of the inlet/outlet ports over each time interval $\sigma$. Such a reactor can be described by a spatially- and temporally-continuous model if we ignore the end-effects of the intermediate sections and apply the boundary conditions at positions that vary in time as stepwise functions with a total period $\theta=N \sigma$. The dimensionless enthalpy and mass balances take the form

$$
L e \frac{\partial y}{\partial \tau}+u \frac{\partial y}{\partial \xi}-\frac{1}{P e_{y}} \frac{\partial^{2} y}{\partial \xi^{2}}=\sum_{k=1}^{N_{r}} B_{k} r_{k}\left(x_{j}, y\right)=f\left(x_{j}, y\right)
$$




$$
\begin{gathered}
\frac{\partial x_{i}}{\partial \tau}+u \frac{\partial x_{i}}{\partial \xi}-\frac{1}{P e_{x i}} \frac{\partial^{2} x_{i}}{\partial \xi^{2}}=\sum_{k=1}^{N_{r}} r_{i k}\left(x_{j}, y\right)=f_{i}\left(x_{j}, y\right), \quad i, j=1,2, \ldots, N_{s} \\
r_{k}=\left(1-x_{k}\right) D a_{k} \exp \left(\frac{E_{k}}{E_{1}} \frac{\gamma_{1} y}{\gamma_{1}+y}\right)
\end{gathered}
$$

The feed position is defined as following

$$
\xi_{\text {in }}=(n-1) \Delta L, \quad t \in[(n-1) \sigma, n \sigma]+k \theta, \quad n=1, \ldots, N ; \quad k=0,1
$$

where $n$ specifies the unit number and $k$ is the cycle number. The outlet position almost coincides with the inlet (in this case it is useful to define the boundaries as $\xi_{\text {in }}=\xi_{b}^{+}, \xi_{\text {out }}=\xi_{b}^{-}$). The commonly accepted Danckwert's $\mathrm{BC}$ are applied:

$$
\begin{gathered}
\xi=\xi_{\text {in }}, \quad \frac{1}{P e_{y}} \frac{\partial y}{\partial \xi}=u\left(y-y^{i n}\right) ; \quad \frac{1}{P e_{x i}} \frac{\partial x_{i}}{\partial \xi}=u\left(x_{i}-x_{i}^{i n}\right) ; \\
\xi=\xi_{\text {out }}, \quad \frac{\partial y}{\partial \xi}=0 . \quad \frac{\partial x_{i}}{\partial \xi}=0 .
\end{gathered}
$$

Here the conventional notation is used: the dimensionless concentrations and temperature $\left(x_{i}, y\right)$ are the dynamic variables, $\gamma_{k}$ is the dimensionless activation energy, $B_{k}$ is the exothermicity of the reaction and $\Delta T_{a, k}$ is the adiabatic temperature rise (ATR) of the $k$-th reaction, respectively:

$$
\begin{array}{r}
y=\gamma_{1} \frac{T-T_{i n}}{T_{i n}}, \quad x_{i}=1-\frac{C_{i}}{C_{i 0}}, \quad \xi=\frac{z}{z_{0}}, \quad \tau=\frac{t u_{0}}{z_{0}}, \quad v=\frac{u}{u_{0}}, \\
\gamma_{k}=\frac{E_{a, k}}{R_{g} T_{i n}}, \quad \Delta T_{a, k}=\gamma_{1} \frac{\left(-\Delta H_{k}\right) C_{i, i n}}{\left(\rho C_{p}\right)_{f}}, \quad B_{k}=\frac{\Delta T_{a, k}}{T_{i n}}, \\
D a_{i}=\frac{z_{0}(1-\varepsilon) A_{i}}{u_{0}} \exp \left(-\gamma_{i}\right), \quad L e=\frac{\left(\rho c_{p}\right)_{e}}{\left(\varepsilon \rho c_{p}\right)_{f}}, \quad P e_{y}=\frac{\left(\rho c_{p}\right)_{f} z_{0} u_{0}}{k_{e}}, \quad P e_{x}=\frac{\left(\rho c_{p}\right)_{f} z_{0} u_{0}}{k_{e}},
\end{array}
$$

Note, that we use arbitrary values $u_{0}, z_{0}$ for the velocity and the length scales in order to ensure that the corresponding dimensionless values $(v, \xi)$ can be varied as independent (control) parameters. In regular simulations we set $u_{0}=u=$ const yielding $v=1$. Typically in our simulations, as well as in practical situations, Le $\gg 1$ (i.e., large solid to fluid heat capacity ratio) and $\mathrm{Pe}_{x}, \mathrm{Pe}_{y} \gg 1$.

Rotating patterns were shown to emerge in such reactors, provided that the switching velocity (i.e., unit length/switching time) and the pattern velocity are matched in a certain way. More precisely two such asymptotes were derived, in which the ratio of the two velocities is either around unity, which we will refer to as slow-switching, or very large, which we will refer to as fast-switching asymptote. The former is the main focus of this review while the latter corresponds to a cross flow reactor in which the feed (reactants) are dispersed along the reactor (i.e. $[30,33,44])$.

Both models can admit, with a generic first-order Arrhenius kinetics and typically large Pe and Le numbers, a rotating-pulse solution with peak temperatures that significantly exceeds the adiabatic temperature rise. Both solutions were corroborated by simulations of finite- $N$ systems that show convergence to the expected asymptotes in realistic system lengths. The asymptotic models enabled us to draw bifurcation diagrams and characterize the properties of emerging solutions.

Between these two asymptotes, the dynamics may become very complex, in a way that can be described as a rotating pulse forced externally and is reminiscent of the forced oscillator problem. 
In order to facilitate the analysis of the emerging patterns with a fixed total period $\theta$ we consider the limiting case of a loop reactor with infinitely many ports $(N \rightarrow \infty, \sigma \rightarrow 0, \Delta L \rightarrow 0)$. In this case the stepwise functions $\xi_{\text {in }}(\tau), \xi_{\text {out }}(\tau)$ can be replaced by continuously varied functions

$$
\zeta_{\text {in }}(\tau)=\xi_{\text {in }}^{0}-V_{\text {sw }} \tau, \quad \zeta_{\text {out }}(\tau)=\xi_{\text {out }}^{0}-V_{\text {sw }} \tau,
$$

where the switching velocity $\left(V_{s w}\right)$ is defined as $V_{s w}=\Delta L / \sigma$. In such a case it is convenient to transform the governing equations (2.1)-(2.3) using a moving coordinate system $\left(\tau^{\prime}=\tau, \zeta=\xi-V_{s w} \tau\right)$ with fixed positions of the inlet and outlet. To simplify the following notation we consider the case of a single reaction yielding

$$
\begin{gathered}
L e \frac{\partial y}{\partial \tau}+\left(v-L e V_{s w}\right) \frac{\partial y}{\partial \zeta}-\frac{1}{P e_{y}} \frac{\partial^{2} y}{\partial \zeta^{2}}=B D a(1-x) \exp \left(\frac{\gamma y}{\gamma+y}\right) \\
\frac{\partial x}{\partial \tau}+\left(v-V_{s w}\right) \frac{\partial x}{\partial \zeta}-\frac{1}{P e_{x}} \frac{\partial^{2} x}{\partial \zeta^{2}}=D a(1-x) \exp \left(\frac{\gamma y}{\gamma+y}\right)
\end{gathered}
$$

The mass balance Danckwerts' BC conditions (see (2.5)-(2.6)) can still be applied in this case. For the energy balance equation, in the case of slow switching $\left(V_{s w} \sim V_{f r}\right)$, the appropriate boundary conditions were shown [37] to be:

$$
\left.y\right|_{\text {in }}=\left.y\right|_{\text {out }}, \quad \frac{1}{P e_{y}}\left(\left.\frac{\partial y}{\partial \zeta}\right|_{\text {in }}-\left.\frac{\partial y}{\partial \zeta}\right|_{\text {out }}\right)=\left.v\left(y-y^{\text {in }}\right) \quad y\right|_{\text {in }}=\left.y\right|_{\text {out }}
$$

The first of conditions (2.11) was derived as a simple combination of the Danckwerts' BC, while the second condition enable the formation of a pulse solution (see the simulation below) and resembles the hot flux supply into the cold section after each switching. It was shown [37] that the solution of the multiport reactor equations (2.1)-(2.6) converges to the asymptotic model equations (2.9)-(2.11) as $N \rightarrow \infty$.

System (2.9)-(2.10) with BC (2.11) corresponds to the limiting case of the discrete port system with $N \rightarrow \infty$ for any (finite) switching velocity. For a case of a fast switching velocity it is possible to derive an asymptotic continuous model that resemble the cross-flow reactor (CFR) in which the reactants are fed along the reactor. While pattern formation in CFR was studied intensively, CFR conditions are established best with reactant supply by a membrane, rather than by fast switching in LR, and fast-switching is not considered in the present review.

\subsection{Slow switching}

Inspection of the problem statement (2.9)-(2.10) shows that this system is quite similar to that of a front propagation in a once-through fixed bed with the switching velocity $V_{s w}$ replaced with the front velocity $V_{f r}$. The main differences between these two problems are the boundary conditions applied. If the length $L$ is sufficiently large, we expect to find in the loop reactor with $V_{s w}=V_{f r}$ stationary solutions in the form of a front or a pulse that rotate in the physical coordinate system. Moreover, we expect that such a 'frozen' rotating pattern can be sustained for a domain of $V_{s w}$ that lies between the combustion front velocity $V_{f r}$ (in a once-through) and the velocity of the thermal front $V_{t h}=v /$ Le, i.e.:

$$
V_{f r}<V_{s w}<V_{t h}
$$

In the following text we set $v=1$ if the otherwise is not stated. Note, equation (1.1) was derived from system (2.9)-(2.10) with $V_{s w}=V_{f r}$, and the ratio of the temperature rises becomes unbound when $V_{s w} \mathrm{Le}=1$. 

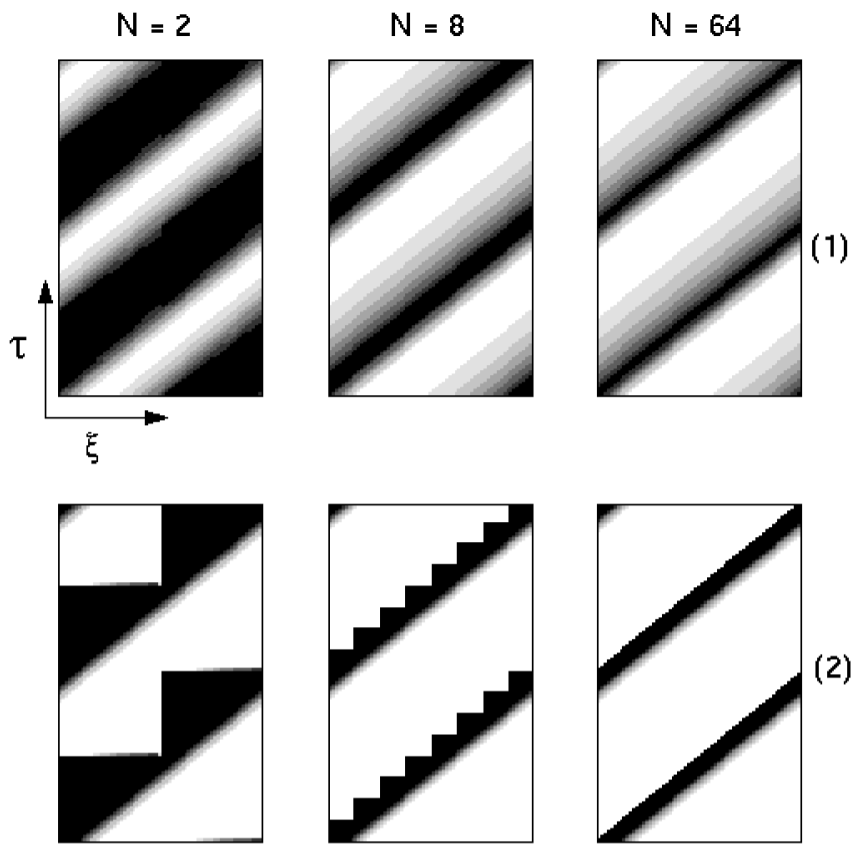

FiguRE 2. Effect of number of units $(N)$ on the spatio-temporal patterns of the temperature (1) and concentration (2). After reference [37]; $V_{s w} / V_{t h}=0.976$, other parameters: $\Delta T_{a d}=$ $50 \mathrm{~K}, u=1 \mathrm{~m} / \mathrm{s}, L=1 \mathrm{~m}, \mathrm{Pe}_{x}=200 ; \mathrm{Pe}_{y}=194 ; L e=800\left(V_{t h}=0.00125 \mathrm{~m} / \mathrm{s}\right)$ and $B_{0}=$ 0.523 .

This expectation as well as the effect of the applied boundary conditions were verified by numerical simulations of the full and the asymptotic models along with approximations of the front velocity and of the temperature rise on the front $\left(\Delta T_{m}\right)$. The approximations for the ideal front propagation in a long system under adiabatical conditions were proposed in reference [18]. The derivation was based on the concept of the narrow reaction zone in an ideal front $[14,41,48]$ with negligible mass diffusion and yields (with $v=1$ ):

$$
\frac{D a}{P e_{y}\left(1-V_{f r}\right)^{2}} \exp \left(\frac{y_{m}}{1+y_{m} / \gamma}\right)=\frac{B}{\left(1+y_{m} / \gamma\right)^{2}}
$$

Equation (2.13) coupled with relation (1.1) form an algebraic system with respect to the front characteristics $\left(T_{m}, V_{f r}\right)$ which can be applied in a domain Le $>1, V_{f r}<\mathrm{Le}^{-1}$.

This system was successfully validated for an adiabatic long reactor [9]. In the following studies the effect of heat losses [15] as well as the effects of finite length BC and of incomplete conversion [21,32] were accounted for.

Since Le $\gg 1$ in catalytic reactors, the domain of "frozen" rotating patterns corresponds to low switching velocities $\left(V_{s w}<\mathrm{Le}^{-1} \gg 1\right)$ when compared with the convective velocity.

Discrete model. A typical rotating-pattern solution of a $N$-unit system with a certain switching velocity $V_{s w}$ in a domain $V_{f r}<V_{s w}<V_{t h}$ is shown in Figure 2. With increasing number of ports $(N)$, while preserving the same switching velocity $V_{s w}$, the stair-like patterns become smoother.

The temperature and concentration profiles with increasing $N(\rightarrow \infty)$ converge to certain limiting solutions (Fig. 3a). This aspect is also illustrated by the plot of the maximal temperature $T_{m} v s$. the inverse number of units (Fig. 3b). The obtained results obviously corroborate the existence of the limiting continuous model (Eqs. (2.9)-2.10)). The matched BC defined by set (2.11) are in a good agreement with numerical simulations. 

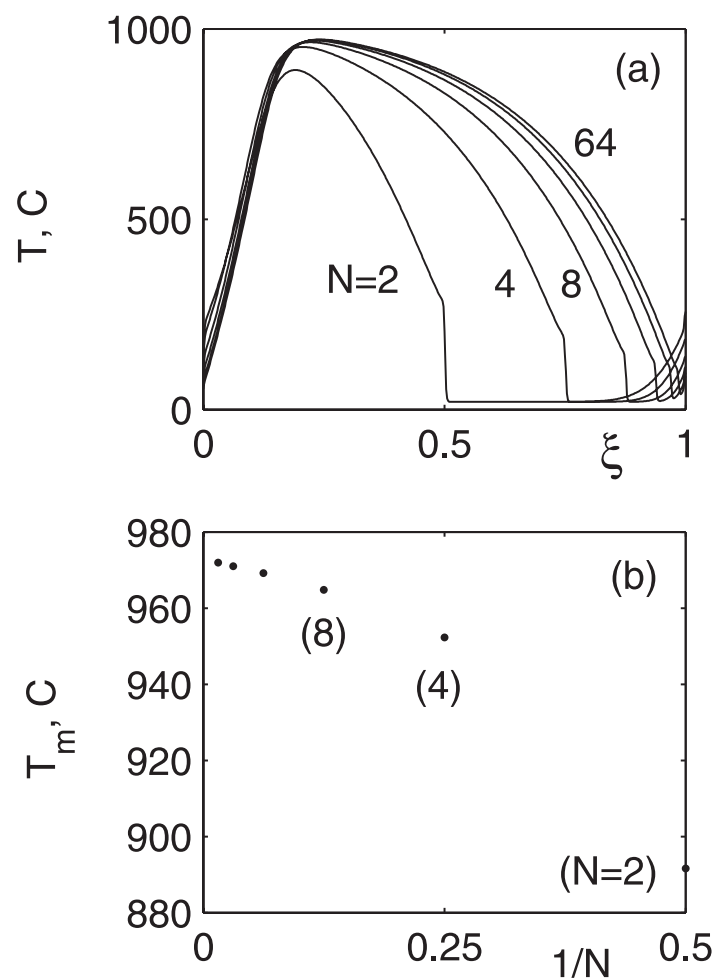

FiguRE 3. Effect of number of units $(N)$ on the spatial temperature profiles (a) and the maximal temperature (b) showing convergence with increasing $N$ to a limiting solution. After reference [37], parameters as in Figure 2.

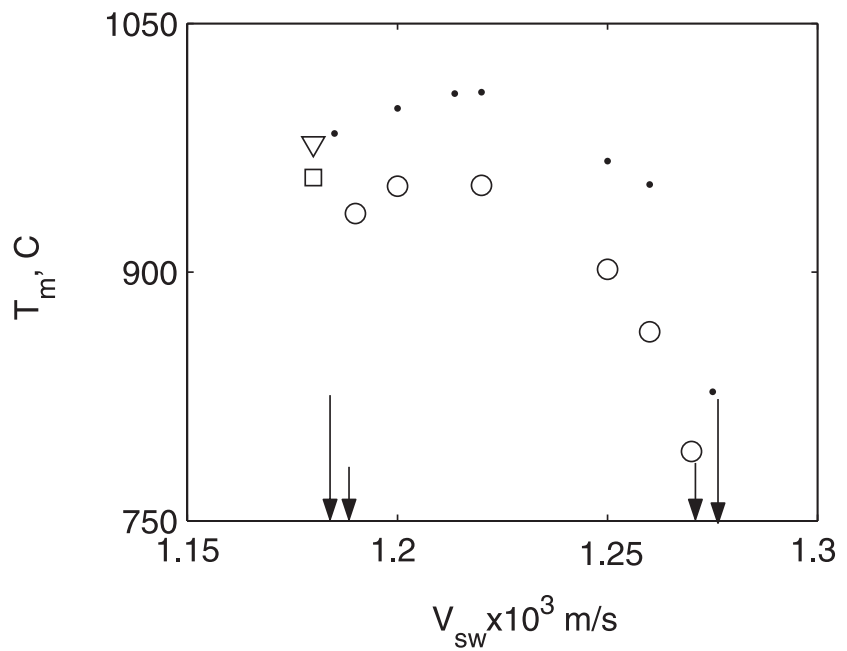

FIgURE 4 . Bifurcation diagrams showing the effect of the switching velocity $V_{s w}$ on the maximal temperature $T_{m}$ in a 4-unit system (open circles) and in the limiting model (dots). The analytically (Eqs. (1.1), (2.13)) and the numerically (in a long system) calculated sets $\left(V_{f r}, T_{m}\right)$ are marked by a triangular and a rectangular respectively. The short and long arrows mark the extinction limits in a 4-unit system and in a limiting model respectively. After reference [37]. 


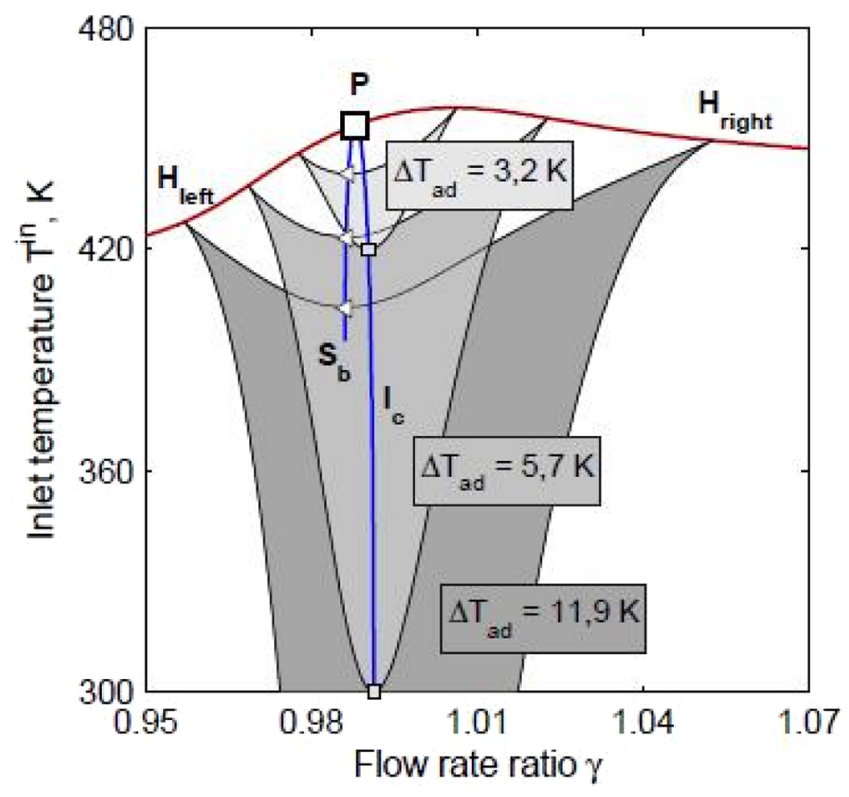

FiguRE 5. Shrinking of the operation window with decreasing the feed concentration and the corresponding singular points. The abscissa is the flow rate ratio $\gamma=V_{s w} / V_{t h}$ (after Ref. [45]).

Frozen traveling waves exist in a narrow domain of parameters (Fig. 4) approximately following condition (2.12) with $0.94<V_{s w} / V_{t h}<1.03$ for the parameters specified in [37] with $V_{t h}=0.00125 \mathrm{~m} / \mathrm{s}$; outside this domain the extinguished solution exists with low exothermicity $\left(B / B_{0} \sim 1, B_{0}\right.$ is a reference value; complex pattern formation with large $B$ is considered below). The limiting model predicts well the results of a discrete section reactor: in the 4 -unit system the patterns exist in a slightly narrower domain $\left(0.95<V_{s w} / V_{t h}<1.02\right)$. Note, that the discrepancy between the discrete and the continuous model decreases with increasing $N$. Also a good agreement between the analytically and the numerically obtained front velocities and the corresponding maximal temperatures is achieved, and completely confirms condition (2.12) which defines the domain of the slow-switching patterns.

Obviously, the locus of $T_{m}$ points is an isolated curve and there exists a branch of unstable states for this domain.

The singularities expected in a loop reactor were characterized $[45,46]$ by plotting the domain of frozen rotating patterns in the plane of the inlet temperature $v s$. the switching velocity $\left(V_{s w} / V_{t h}\right.$, denoted as 'Flow rate ratio' $\gamma$ in Fig. 5). Let us follow the domain for the adiabatic temperature rise (denoted also as ATR or $\Delta T_{a}$ ) of $11.9 \mathrm{~K}$ (denoted by dark gray): It is organized around $V_{s w} / V_{t h}=1$ with three branches of forced periodic solutions (upper-stable, intermediate-unstable and lower-stable and inactive) in which the top two are organized as an isolated curve. With $T_{i n}>400 \mathrm{~K}$ the isola coalesces with the inactive branch and splits to form a 'mushroom' shape. Further increase in $T_{i n}$ leads (at point $S b$ ) to formation of an $S$-shaped and an inverseshaped hysteresis loop. At higher $T_{i n}$ the two hysteresis loops disappear at the points denoted $H_{\text {right }}, H_{\text {left }}$. With decreasing $\Delta T_{a}$ (i.e., more dilute streams) the two hysteresis points approach each other until their disappearance at $\Delta T_{a}<3.2 \mathrm{~K}$. It was shown [43] that the minimal concentration (expressed as ATR) is below $20 \mathrm{~K}$ at feed temperature of $450 \mathrm{~K}$. In this case the result is not meaningful technologically, but the unfolding of the singular point with other parameters may be of interest in the general case.

\subsection{Intermediate switching}

The pattern need not be simply periodic and rotating. A rich plethora of patterns depending both on the switching velocity $V_{s w}$ and the number of units $N$ was obtained with $V_{s w} / V_{t h}$ that varies between the two 


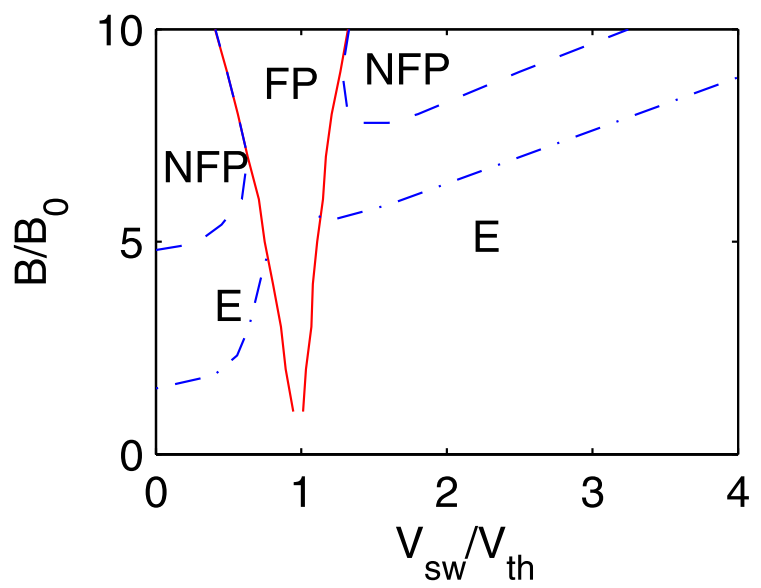

Figure 6. Bifurcation map of the asymptotic loop reactor model in the $\left(B / B_{0}, V_{s w} / V_{t h}\right)$ plane showing domains of frozen patterns (FP, i.e. steady in a moving coordinate, solid lines), and of non-frozen patterns (NFP, periodic or aperiodic in a moving coordinate, dashed with $L=1$ and dashed-dotted with $L=8$ ) and of extinguished (E) solutions. After [31], other parameters as in Figure 2.

asymptotes. This is of academic interest, as well as of practical interest since the main drawback of the LR is the very narrow window of switching velocities that sustain a stable 'frozen' solution that exists if the switching and the pulse velocity are synchronized. An active (ignited) solution may be sustained in the form of complex dynamic patters. The authors showed [31] the existence of many 'finger'-like domains of complex frequency-locked solutions that allow to significantly extend the operation domain, rendering the LR scheme more attractive for practical implementation.

Asymptotic model: Figure 6 portrays the domains of existence of ignited solutions in the plane of exothermicity $\left(B / B_{0}, B_{0}\right.$ is a reference value) vs. $V_{s w} / V_{t h}$. The system also attains an extinguished solution for the whole domain, while the ignited state can be sustained with a proper choice of initial conditions. The domain of 'frozen' patterns (FP, i.e., steady in a moving coordinate) forms a cusp-like structure that expands with increasing the feed concentration or the adiabatic temperature rise $(B)$ and shrinks with increasing convective velocity $v$ (not shown here). To understand the gain made with the Loop Reactor note that it can sustain rotating patterns with $B / B_{0} \sim 1\left(V_{s w} / V_{t h} \sim 1\right)$ while a once-through fixed bed (i.e., $\left.V_{s w}=0\right)$ requires an order of magnitude higher exothermicity $\left(B / B_{0} \sim 13\right)$. Non-frozen rotating patterns (NFP, i.e., periodic or aperiodic in a moving coordinate) exist in two domains adjacent to the FP domain on its two sides as is shown in Figure 6.

A typical temperature pattern transformation, upon varying the switching velocity within both the NFP and the FP domains show the temperature patterns (Fig. 7 ) in the $(\tau, \zeta)$ plane (i.e. in a coordinate system moving with the switching velocity $V_{s w}$ ). The solution presents right- and left-propagating waves for the left- and right NFP domains (Fig. 7a, b, e, f), respectively, and stationary solutions within the FP domain (Fig. 7c, d). The bifurcations in Figures 6 and 7 from the periodic solutions (Frozen Pattern) to the inactive state are associated with hysteresis and are likely via a saddle-loop bifurcation of limit cycles (coalescence of stable and unstable limit cycles). The FP bifurcation to NFP occurs via a Hopf bifurcation (in the moving coordinate). A better characterization appears at the end of the following section.

Discrete unit model: The analysis was extended to the practical case of a small number of units $(N)$. In this case the system can exhibit both simple solutions, rotating with a velocity close to the 'ideal' front velocity $V_{f r}$, and complex oscillations of 1:n form defined by the ratio of the pulse rotation to the feed switching velocities (Fig. 8). Following the classification employed for the asymptotic model we refer to these solutions as quasi-frozen patterns (QFP) and quasi non-frozen patterns (QNFP). We add the term 'quasi' as we based the characterization on the figures like Figure 9 and not on precise measurements. 
(a)

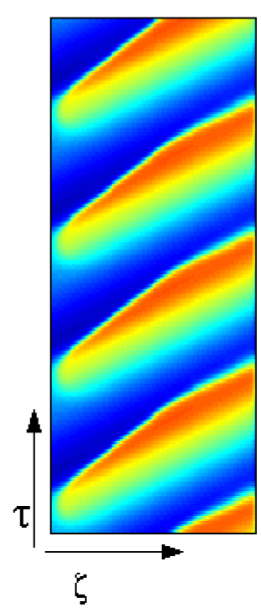

(b)

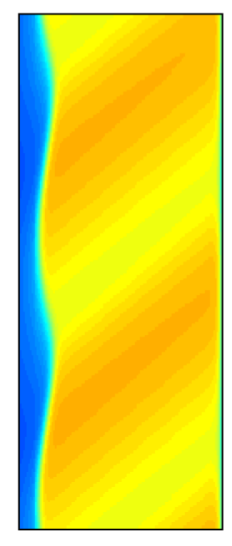

(c)

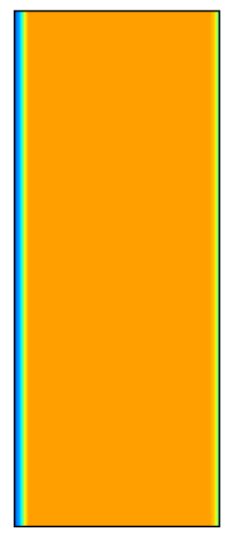

(d)

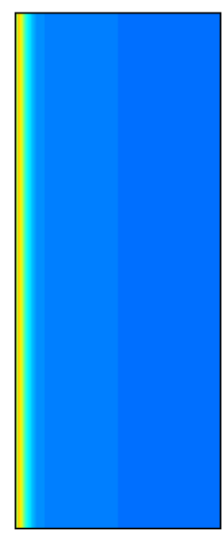

(e)

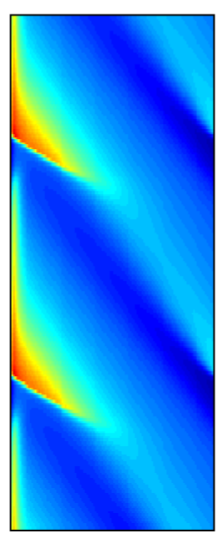

(f)

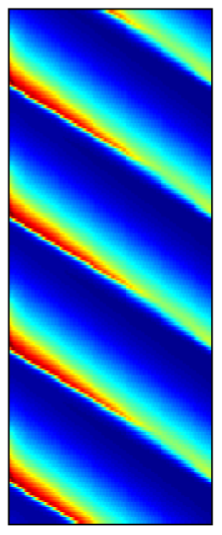

FiguRE 7. Typical transformation of the temperature patterns of the asymptotic LR model in a moving coordinate system $(\zeta, \tau)$ with the switching velocity: Shown are non-frozen rotating patterns in NFP1 domain $\left(V_{s w} / V_{t h}=0, \mathrm{a} ; 0.4, \mathrm{~b}\right)$, frozen patterns $\left(V_{s w} / V_{t h}=0.48, \mathrm{c} ; 1.25, \mathrm{~d}\right)$ and non-frozen rotating patterns in the NFP2 domain $\left(V_{s w} / V_{t h}=1.36\right.$, e; $\left.2.4, \mathrm{f}\right), B / B_{0}=10$, $L=1$. After reference [31].
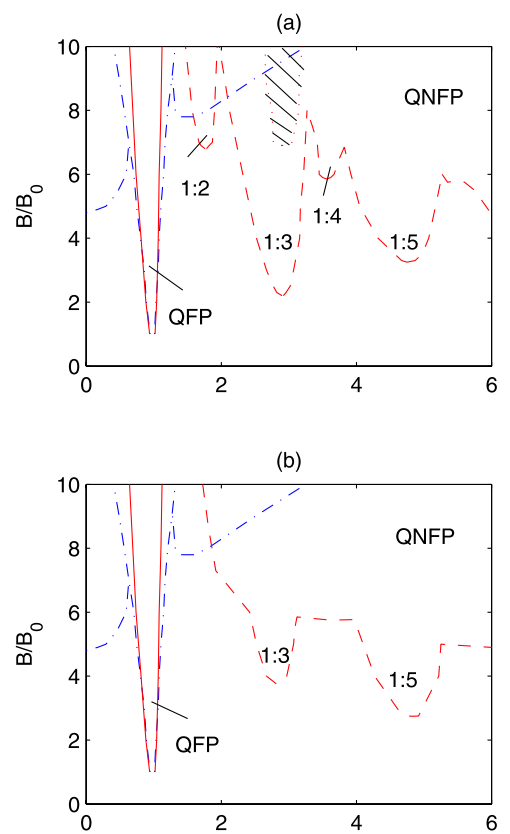

Figure 8. Typical structure of the finger-like domains of the two-unit (a) and four-unit (b) LR model in the $\left(B / B_{0}, V_{s w} / V_{t h}\right)$ plane showing domains of quasi-frozen (QFP, solid lines) and quasi non-frozen (QNFP, dashed lines) solutions. Dashed region shows subdomain of the $(1: 3)_{3}$ solution. Dashed-dotted lines mark the boundaries of the FP and NFP domains of the asymptotic model. Numbers 1:n mark the type of the frequency locked solutions (after [31]). 
(a)

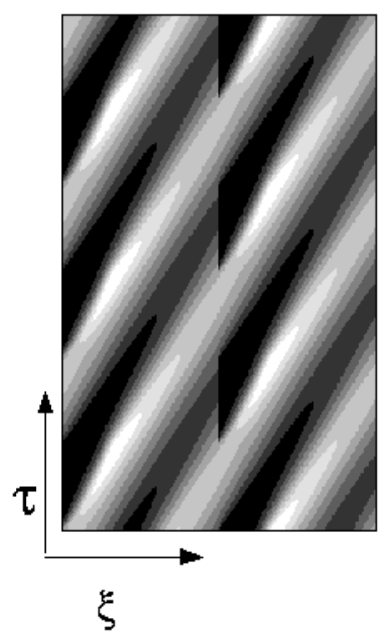

(b)

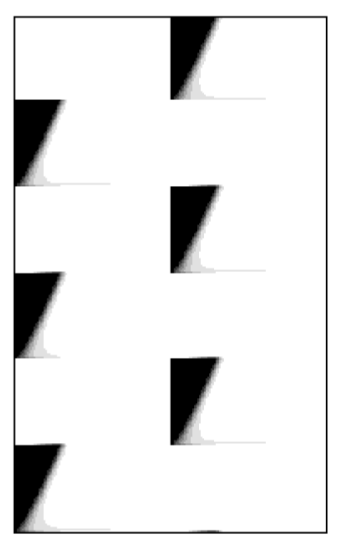

FiguRE 9. Typical regular $(1: 3)_{3}$ solution of a two-unit loop reactor showing the temperature (a) and the conversion (b) patterns during one pulse rotation period in a stationary coordinate system $(\xi, \tau) . B / B_{0}=8, V_{s w} / V_{t h}=3$; other parameters as in Figure 2. After reference [31].

For the QNFP with a single pulse the total period of oscillations $\left(\theta_{t o t}\right)$ coincides with the period of the pulse rotation $\left(\theta_{f r}\right)$. Such patterns repeats itself every $N \times n$ switches (for example, 6 in the case of the 1:3 solution with 2-unit reactor) and admits the symmetry:

$$
\varphi \xi, \tau)=\varphi(\xi+\Delta L, \tau+n \sigma), \quad \xi \in[0, \Delta L], \quad \tau \in[0, n \sigma]
$$

where $\varphi$ is either of the state variables $y$ and $x$ and $\Delta L=L / N$ (with increasing $N$ the variety of possible regular solutions becomes larger).

Typical maps showing 'finger'-like domains of patterned states in the $\left(B / B_{0}, V_{s w} / V_{t h}\right)$ plane for systems with $N=2$ and 4 are shown in Figure 8. The QFP subdomain and the 'finger'-like QNFP domains are separated by gaps of extinguished states at low $B$. For large $B$ the QNFP domains merge to yield a continuous domain with respect to varying $V_{s w}$. The QFP domain for a systems with a certain $N$ is enclosed within the corresponding domain of the asymptotic model (at $V_{s w} / V_{t h}=O(1)$ ). The boundaries calculated for a two- and a four-unit systems almost coincide and expand toward the limiting model with larger $N$.

Within the QNFP domains the system exhibits a spatially and temporally varying pulse rotating with a velocity close to the 'ideal' front velocity. For a set of parameters employed in this study we detected (with $N=$ 2) large domains of 1:n solutions with odd $n=3,5,7$ and relatively small subdomains of even $n$, that exist only for large $B$.

In addition to complex QFP (1:n) solutions considered above we detected (at large $B$ ) multiwave patterns of $(1: n)_{k}$ type (i.e. $k$ pulses coexist and move through the system, while the ratio of the pulse rotation and the switching velocities is 1 to $n$ ). A typical (1:3) 3 pattern exhibits three ignited pulses along the reactor (Fig. 9). Its subdomain in the parameter plane is shown in Figure $8 \mathrm{a}$ as a dashed region. Note that such patterns can be both periodic and aperiodic with time and space.

\subsection{Experimental demonstration}

Sustained rotating frozen patterns were demonstrated with ethylene oxidation as a model reaction [22, 23]. This reaction can be carried out at a relatively low temperature $(\sim 200 \circ \mathrm{C})$, and our group proceeded to demonstrate this concept with methane combustion which requires a maximal temperature of $600 \circ \mathrm{C}$ or so [21]. The 

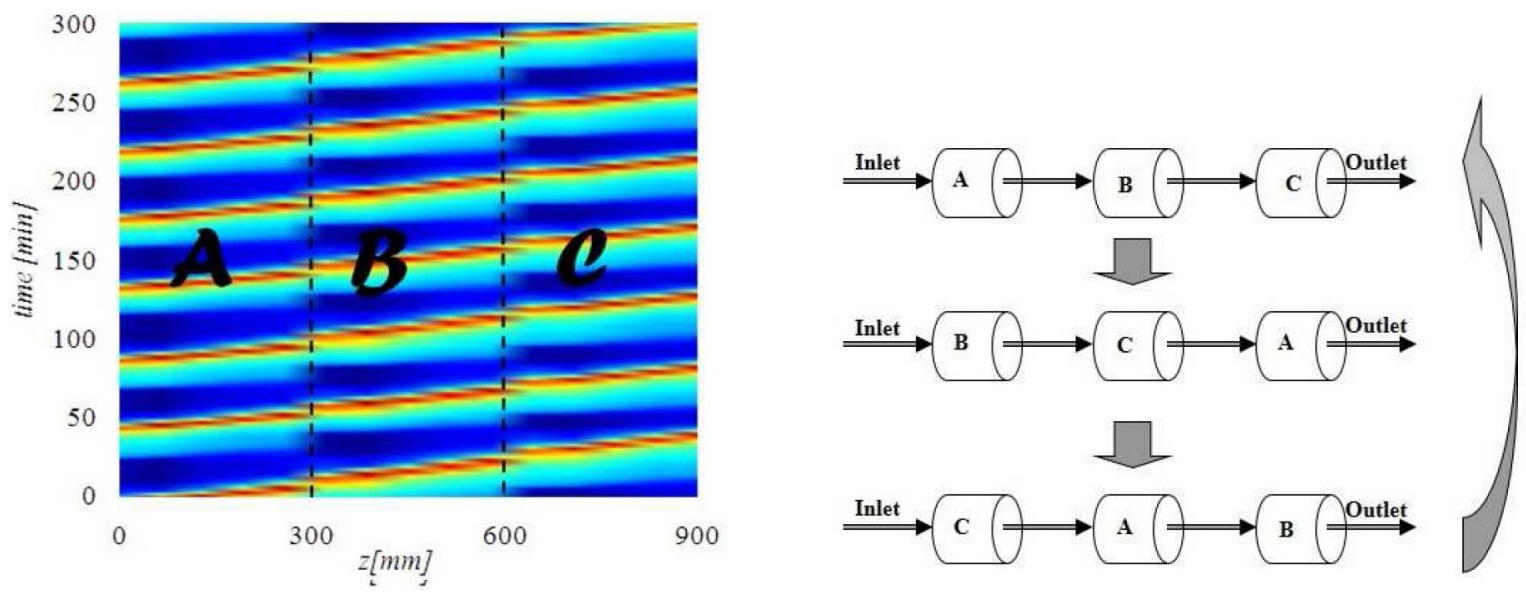

FiguRE 10. (left) Spatio-temporal color-scaled temperature profile, measured during methane combustion in a 3-unit system, and presented in the time-space plane (air flow $25 \mathrm{~L} / \mathrm{min}$ with 19,400 ppm methane in the feed); (right) schematic description of the process. After reference [21].

experimental system incorporates three fixed-bed reactors arranged in the form of a loop. The three reactors, each bent in its middle to form a hexagon-shaped system, and each $30 \mathrm{~cm}$ long, are packed by spherical catalytic particles $\left(0.5 \% \mathrm{Pt} / \mathrm{Al}_{2} \mathrm{O}_{3}\right.$ in $2-4 \mathrm{~mm}$ diameter). Fifteen thermocouples were placed along the three reactors.

The control method applied was as follows: for two consecutive reactors, the feed is switched from one reactor to the following when the temperature measured at the entrance of the second reactor reaches a certain threshold $T_{s w 2}$ and the temperature at the entrance of the first reactor drops below $T_{s w 1}$. This issue is discussed further in the next section.

A typical periodic temperature profile of the system fed with methane in air, under control, is shown in Figure 10a in the time-space plane using a color-scale for temperature. The abscissa denotes the distance from feed point (the 3 reactors are denoted by $\mathrm{A}, \mathrm{B}, \mathrm{C}$ and the operation is schematically described by the right plate). The pulse propagation is evident from the continuous, almost linear, red stripe with a slope, i.e., the front velocity, of $\sim 2.5 \times 10^{-3} \mathrm{~m} / \mathrm{s}$.

The leanest stream that can sustain the patterned state for the conditions of Figure 10 was experimentally determined to be of $8000 \mathrm{ppm} v s$. 33,000 ppm in a once-through reactor. Decreasing the feed flow rate causes a decline in the maximal temperature (Fig. 11a) until extinction occurs (at $u_{0}=0.225 \mathrm{~m} / \mathrm{s}$ ). The pulse velocity $\left(V_{\text {pul }}\right)$ depends mainly on the flow velocity and on Lewis number (Le). As expected the obtained switching velocity increases with the flow velocity (Fig. 11b).

The analysis of the effects of the heat loss and of the reactor size on the leanest stream (expressed in terms of the adiabatic temperature rise $\Delta T_{a}^{l i m}$ ) that will sustain the operation shows the following [21]: For an adiabatic infinitely long reactor $\Delta T_{a}^{l i m} \rightarrow 0$ while for a finite reactor $\Delta T_{a}^{l i m}$ scales as $\left(1+\mathrm{Pe}_{y} / 4\right)-1$ with $\mathrm{Pe}_{y}$ defined via the reactor length (Eq. (2.7)); the heat loss increases this limit by $\left(\beta P e_{y}\right)^{1 / 2}$ where $\beta=2 U z_{0} /\left(r_{w}\left(\rho c_{p}\right)_{f} u_{0}\right)$ is the dimensionless heat transfer coefficient. Thus, a good design of a LR will aim to decrease the conductivity $(k)$, that affects $\mathrm{Pe}_{y}$ ) and the radial heat-transfer coefficient $(\beta)$ while increasing throughput (i.e., the convective velocity, $\left.u_{0}\right)$ and the reactor length. Note that in practice it is almost impossible to achieve adiabatic conditions.

In another experimental study [47] a two-unit loop reactor for catalytic propene complete oxidation was tested using a control scheme described below. Figure 12 describes a long record of temperatures measured by a single thermocouple in each unit and of the switching interval, in response to changes in flow rates $(0-45 \mathrm{~h})$ or in response to changes in feed concentration (45-84 h). Full conversion was achieved throughout these changes. Note that switching are slower upon increasing of the flow rate or of the feed concentration. 

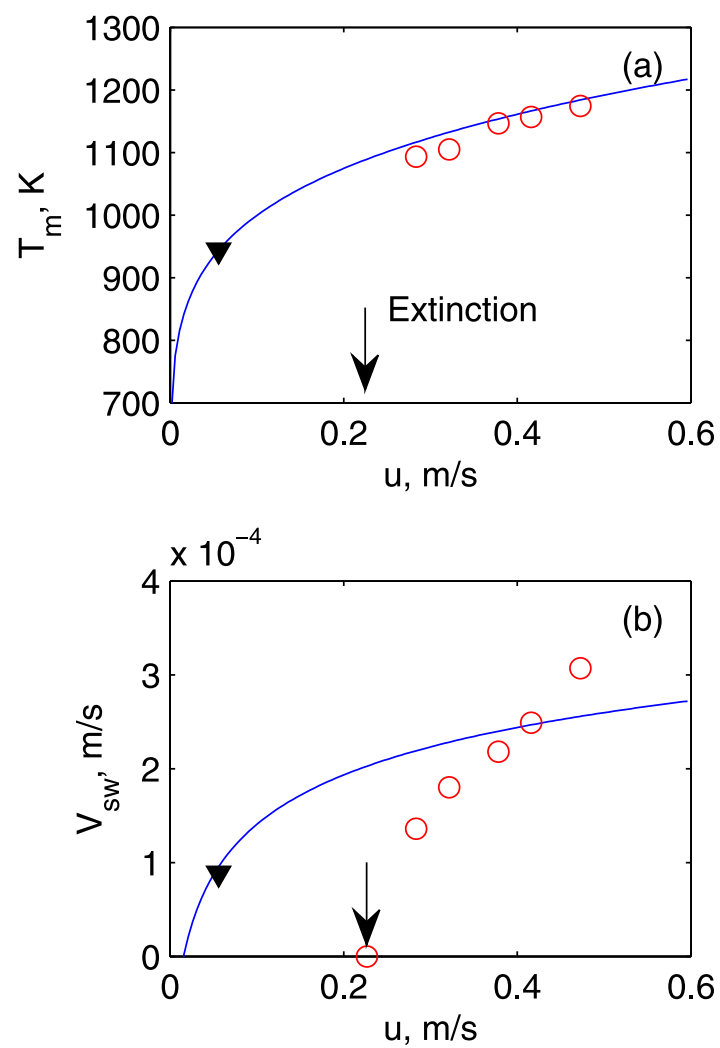

FiguRE 11. The effect of the feed flow rate $(u)$ on the maximal temperature (a) and on the corresponding switching velocity (b) during methane combustion. Experimental data and approximations (Eqs. (11) and (12) in Ref. [21]) are marked by open circles and lines, respectively. Triangular mark the limiting approximated value. $\left(L=0.9 \mathrm{~m}, T_{s w 1}=T_{s w 2}=875 \mathrm{~K}\right.$, feed of 20,000 ppm methane in air. After reference [21].

Three different controllers were investigated [47] and the author concluded that the most effective approach, requiring the minimum number of temperature sensors, is to install a thermocouple at the end of each reactor and to switch when the temperature at the end of the fed reactor becomes lower than a prescribed set point. Its feasibility is evident from Figure 12.

\subsection{Operating with large feed leaps}

A series of works considered the behavior of a LR with leaps in the feed position of several units $\left(n_{s}>1\right)$, claiming that the designs considered above (i.e., single unit leap, $n_{s}=1$ ) yields narrow domains of operation and requires tight control $[24,25]$. In the cited references the shift by one unit in a downstream direction (considered in the previous section) is referred as strategy 1 , while the shift by one unit in an upstream direction is referred as strategy 2 .

Mancusi et al. [24], studying a reversible exothermic reactions, in a 3-unit nonadiabatic system, compared the two strategies: The features of strategy 1 are similar to those described above. With strategy 2 (feed port leaping back one unit) the isola of $T$-periodic solutions exists as well. However, since in the adiabatic systems the front velocity is the same in both strategies, the time between switches is $(N-1)$ times larger in strategy 2 than in strategy 1. For non-adiabatic system the cooling effect leads to decreasing of this ratio as well as the 

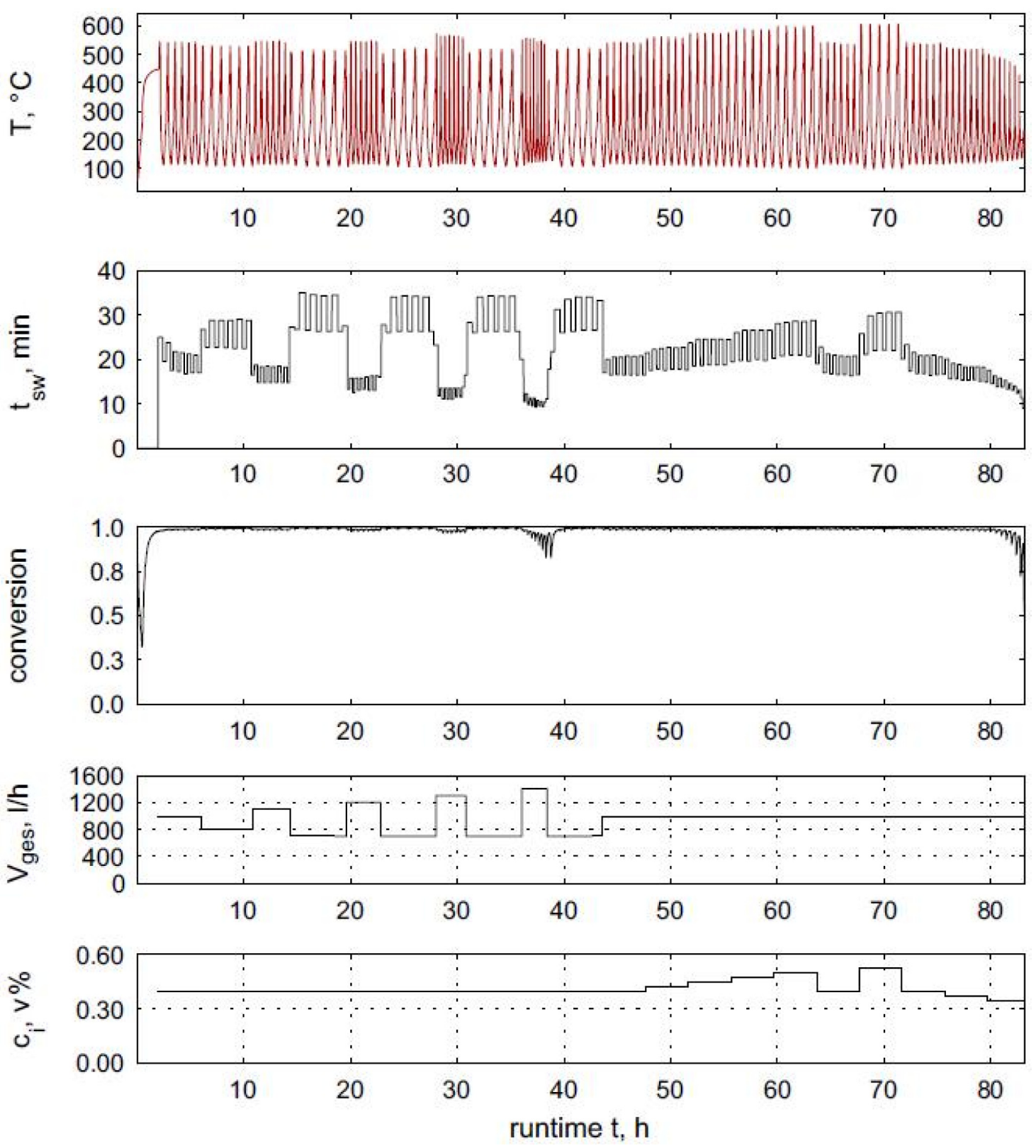

FIgURE 12. Experimental results of a 2-unit reactor in response to input steps in the total flow rate in the first part (up to $45 \mathrm{~h}$ ) and in response to steps in the feed concentration in the second part. The temperature of one reactor is shown in the upper chart, below are the controlled switching time, the conversion and the corresponding inputs of the flow rate and the feed concentration. After reference [47].

decreasing of the maximal temperature. The transition from periodic to multi-periodic behavior was identified as a Neimark-Sacker bifurcation $\left(\tau_{N S} \geq n_{s} / V_{t h}\right)[24]$.

The comparison of the two switching strategies was extended in reference [25] for a 4-unit adiabatic system. Figure 13 shows a schematic representation of the domains of existence of symmetric and asymmetric periodic 

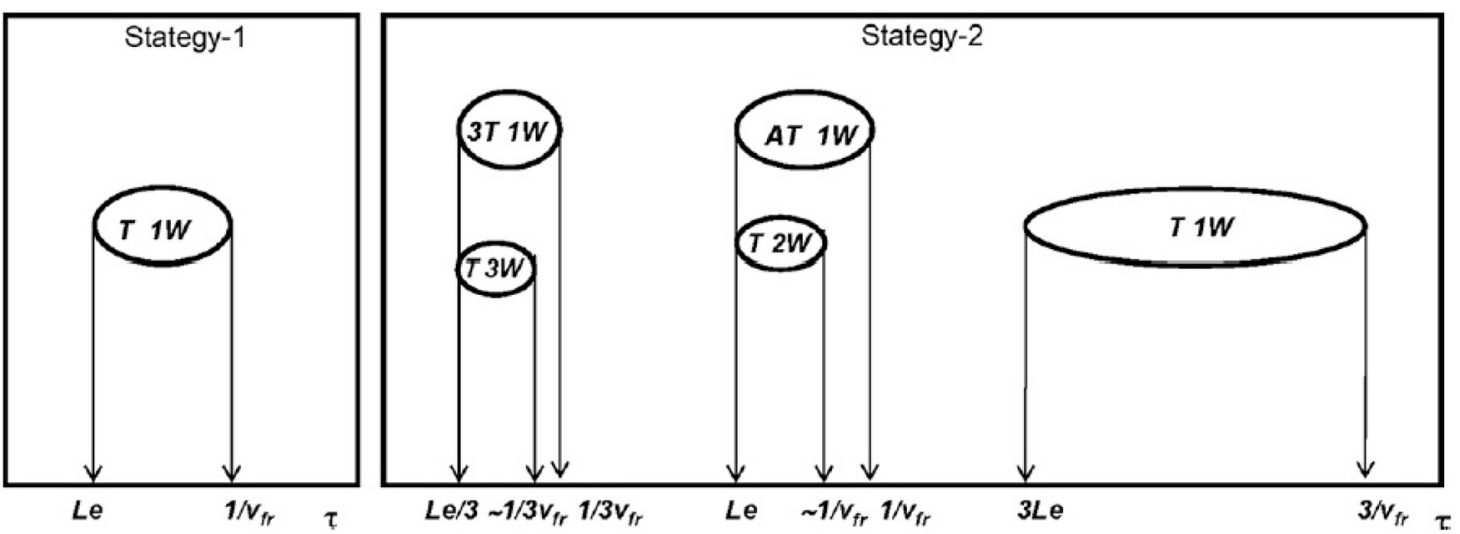

FIGURE 13. Schematic representation of the domains of existence of symmetric and asymmetric periodic regimes. $1 W$ and $n W$ indicate single-wave and multi-wave temperature patterns; $A$ denotes asymmetric regimes and is not reported for symmetric regimes; $n T$ and $T$ are used for $n T$ - and $T$-periodic regimes. After reference [25].

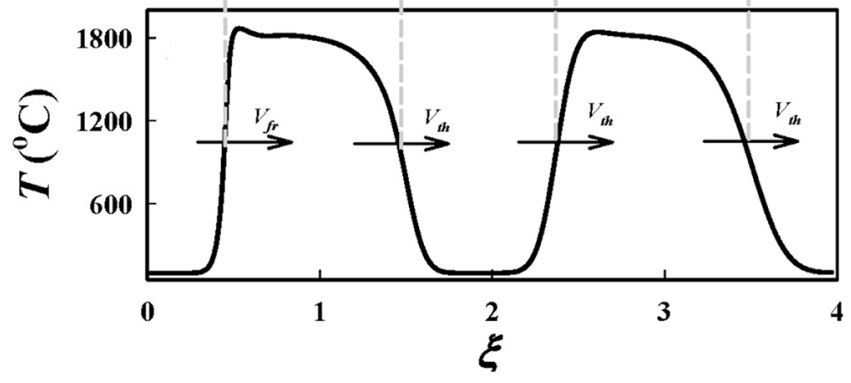

FigURE 14. The temperature profile for a $T$-periodic two-wave solution with $n_{s}=3$ and $N=4$. After reference [3].

regimes vs. the inverse switch velocity $(\tau)$. For $n s=-1$ (or $n_{s}=3$ for a 4 -unit scheme, strategy 2 ) the authors have shown several effects: (i) the number of intervals of $T$-periodic regimes increases with decreasing $\tau$ or increasing the switching velocity (Fig. 13); (ii) the wave may incorporate several thermal fronts, as well as one reaction front ( $n W$ indicate a multi-wave pattern), and (iii) multiple patterns may coexist, depending on initial-conditions. We expect that the plethora of patterns extends with increasing $n_{s}$.

Note that the same features were portrayed for the case $n_{s}=1$ in Figures 6 and 8 . While the domain of multi-periodic solutions may be wider than that of periodic one, suggesting that the control may be more robust, the data in Figure 8 suggests that the $T$-periodic domain may be operated with smaller adiabatic temperature rises. Also, it seems that the control of such multi-fronts waves patterns may be quite challenging. These points deserve further investigation.

Analysis by Altimari et al. [4], based on a geometrical approach of systems, showed infinitely many domains of $T$-periodic regimes corresponding to thermal wave trains with various number of waves. Analytical approximations to the stability limits and the spatiotemporal pattern of these solutions were derived for the case of a fast irreversible exothermic reaction. In such a case, a narrow reaction zone is formed traveling at an approximately-constant velocity $V_{f r}<V_{t h}$ followed by only purely thermal fronts with velocity $V_{t h}$.

Figure 14 shows [3] a traveling wave simulated with $N=4, n_{s}=3$ (or $n_{s}=-1$; the two cases are identical in adiabatic systems), composed of 4 fronts, traveling at $V_{f r}$ (the 1st front) followed by 3 thermal fronts. The 
authors derived [3] relations between $V_{s w}, V_{t h}$ and $V_{f r}$ that will sustain waves with a desired number of fronts. This analysis was generalized for any $n_{s}$ and $N$.

\section{Control}

Control aimed at stabilizing a moving pattern has only been rarely addressed in the literature. A few studies were devoted to develop control strategy that will sustain the propagating pulse solution in the slow-switching mode. The main problems associated with this task are: (i) a narrow domain of parameters in which the desired pulse solution exists, (ii) instability at the boundaries of this domain, (iii) fluctuations in the operation conditions (feed concentration, flow rate), and (iv) uncertainty of the parameters. To overcome the problem of reaction extinction several control actions have been proposed in the literature.

We note here especially the control [6] which switches the feed position when the temperature measured at the sensor located at the following unit ascends beyond a certain threshold and the sensor of the fed unit reads a temperature below another threshold. This approach had been successfully experimentally implemented [21, 22]. Three control concepts were computationally compared [47] and the best one, as outlined in Section 2.4, was used for the control in the experimental studies.

The analysis of dynamics and stability of the network with any numbers of units [38,39] allowed to develop a systematic switching feedback control strategy to stabilize rotating pulses. The switch velocity was used as a control parameter, since it is easily manipulated. In the first stage of analysis they considered the following control law

$$
\frac{\mathrm{d} V_{s w}}{\mathrm{~d} \tau}=-k\left(y\left(\zeta^{*}, V_{s w}\right)-y^{*}\right)
$$

where $V_{s w}$ is responding to measured temperature at a distance $\zeta^{*}=\xi^{*}-V_{s w} \tau$ (i.e., the sensor position is fixed in a moving coordinate). The switch velocity is evaluated at every step on-line using the discrepancy between the temperature at the front of a pulse and the set point. This allowed to study the stability of control in moving coordinates. In the second stage they considered the problem with sensors in fixed coordinates. Note that the system does not have a steady solution that can be used for the linear analysis. It has a periodic solution which can be approximated as a 'frozen' solution when portrayed in a moving coordinate. Analytical results for a limiting many-unit system were presented and applied for the design a system with finite number of units.

A simplified, one-variable, model obtained under the realistic assumptions of Le $\gg 1, \mathrm{Pe}_{x} \gg \mathrm{Pe}_{y} \gg 1$, was derived, that take the form

$$
L e \frac{\partial y}{\partial \tau}+v \frac{\partial y}{\partial \xi}-\frac{1}{P e_{y}} \frac{\partial^{2} y}{\partial \xi^{2}}=D a\left[B-\left(v-V_{s w} L e\right) y+\frac{1}{P e_{y}} \frac{\partial y}{\partial \xi}\right] \exp \left(\frac{\gamma y}{\gamma+y}\right)
$$

The maximal temperature rise in this simplified model is $\Delta y_{m}=B /\left(v-V_{s w}\right.$ Le) as in the general case (1.1) and $\Delta y_{m}=B$ when $V_{s w}=0$ as in the detailed model. This model allows to derive stability conditions for the control scheme and to suggest a control scheme for a realistic system with one sensor, at fixed position in lab coordinates, in each unit. Thus, the control should automatically adjusts $V_{s w}$ to assure its value within the range (2.12).

One example of the effectiveness of this control, in a four unit LR of different Le (i.e., different solid heat capacity), namely 700 (1st), 800 (2nd), 900 (3d) and 800 (4th) is presented in Figure 15. Recall that the frozen rotating pulse solution is stable in a narrow domain $\left(0.94<V_{s w} / V_{t h}<1.03\right.$ for the parameters used), while the thermal front velocities vary $\left((1.43,1.25,1.11\right.$ and 1.25$) \times 10^{-3} \mathrm{~m} / \mathrm{s}$ in the 4 units $)$.

An analogous control structure was employed for the robust stabilization of the single-wave solution of a network carrying out methanol synthesis [35]. A proportional controller and a proportional-derivative controller were implemented to update the switching time in response to the difference between the axial position reached 


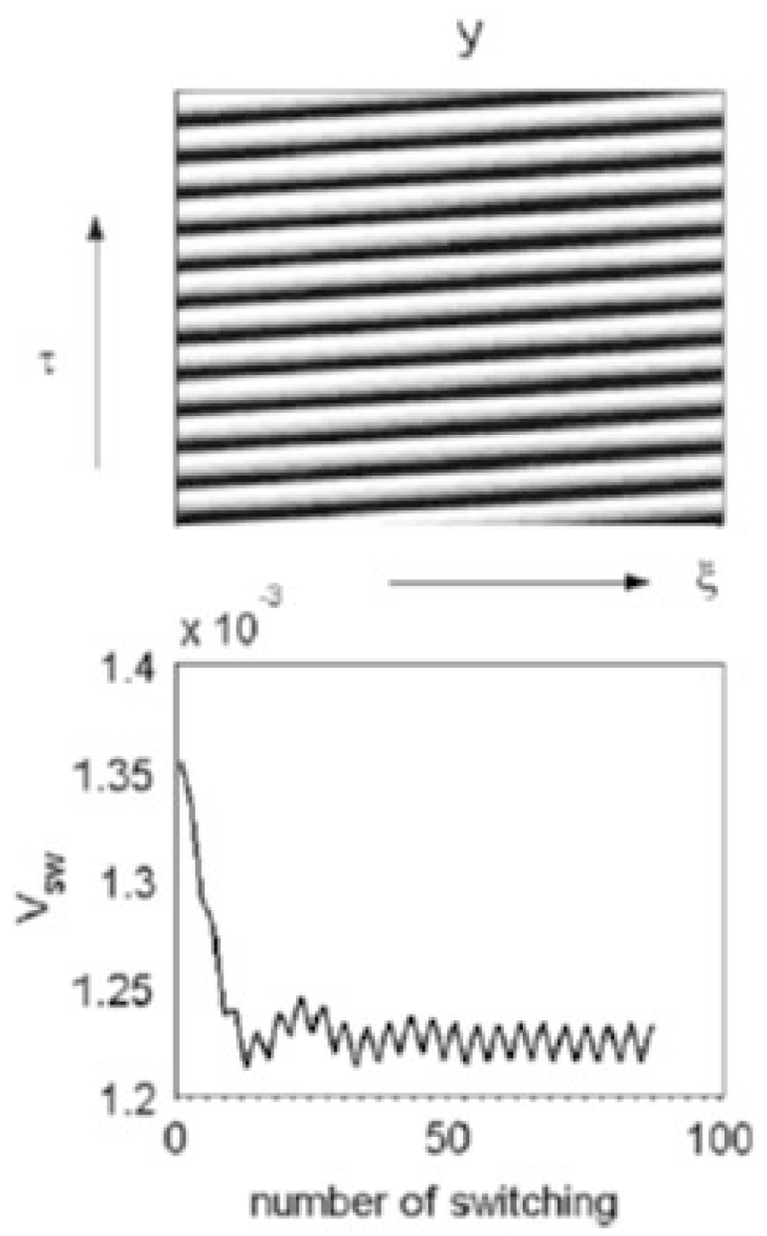

FIGURE 15. Testing the effectiveness of control law on a 4-unit loop reactor when Le varies from the 700 in first unit to 800 in second, 900 in third and to 800 in fourth unit. Figure shows spatiotemporal gray-scale patterns of the dimensionless temperature (top) and dynamics of the $V_{s w}$ response $v s$. the number of switching (bottom); initially $V_{s w}=1.355 \times 10^{-3} \mathrm{~m} / \mathrm{s}$. After reference [38].

by the reaction front at the end of each cycle, and a set-point value. The set point was updated during each cycle based on the online estimation of the reaction-front velocity.

In a study addressing the problem of stabilizing wave-train solutions [5] the proportional control structure proposed in [34] was employed and a controller design procedure enforcing the stability of wave-train solutions was described. The implemented control was adaptive as the set point was updated during each cycle based on the estimated value of the reaction front velocity.

Control strategy was extended [5] for the thermal wave trains, produced by shifting the feed port by several units (see Sect. 2.4). Infinitely many domains of thermal wave trains were demonstrated to exist for any value of shift steps and any number of reactors composing the network, and analytical approximations were derived for the stability boundaries of the predicted solutions based on geometric analysis of the spatiotemporal temperature pattern.

Other advanced approaches, such as the use of observers or soft sensors [10, 13] for forced unsteady-state reactors, have been proposed to control a single traveling temperature wave. 


\section{Multi-SPECIES SYSTEM}

Practical applications of loop reactors will be aimed to obtain a high-conversion combustion of a mixture of hydrocarbons (HC's or VOC's). Design then should aim at a temperature sufficiently high to assure that all reactions will proceed at a reasonable rate. But it is not clear whether the slowest reaction will benefit from the heat liberated. More specifically, we want to determine whether the extinction of that slow reaction (combustion of the less reactive VOC) in a mixture will occur under conditions (i.e., feed concentration and flow rate) that are different than those in a single reaction system. The dynamics and design implications of a bi-species (methane or ethane with ethylene) and of a multi-species combustion was studied by the authors [29].

Qualitative analysis of a binary mixture shows that if two fronts are established and $V_{f, 2}>V_{f, 1}$, i.e., when the high temperature component front propagates faster, the fronts separation increases with time. (Indexes 1 and 2 denote the faster reaction (i.e., of lower maximal temperature, $T_{m}$ ) and the slower (of the higher $T_{m}$ ) reaction components, respectively.) In an opposite case with $V_{f, 1}>V_{f, 2}$ a locked front is obtained as the conversion fronts are coupled via the energy balance and behave as a single front. The proposed approximations were derived for an infinitely long system, while assuming a full conversion of both components, and are summarized as following:

'Locked' solutions. The maximal front temperature in the mixture $\left(T_{m}^{l o c}\right)$ follows the approximation obtained for a single component (2.13) using the activation energy and the pre-exponent factor of the slower reaction $(i=2)$. The front velocity is affected by the sum of the adiabatic temperature rises of both components.

Separated fronts. The parameters of each front can be estimated separately using the one-component approximations assuming that the cooling effect does not lead to significant decline of the temperature ahead of the first front and accounting for the fact that the temperature of the trailing $\left(1^{s t}\right)$ front yields the inlet temperature for the leading one.

Therefore, the approximations of the maximal temperature for both types of solution yield similar results and can be presented by a single component approximation for the second species. The front velocities of the multicomponent mixture are markedly different: For the 'locked' solution $V_{f}^{\text {loc }}$ depends on the total ATR $\left(\Delta T_{a, t o t}=\Delta T_{a, 1}+\Delta T_{a, 2}\right)$ explicitly, while in a separated front solution the leading front velocity exhibits an essentially nonlinear dependence on both $\Delta T_{a, i}$.

The boundary between the locked' and the separated front structures. At the boundary all three velocities should be equal $V_{f, 1}^{\text {sep }}=V_{f, 2}^{\text {sep }}=V_{f}^{\text {loc }}$. The analysis revealed that the system behavior is dictated by the fraction of the low reactivity (high $T_{m}$ ) component in the mixture that can be expressed via the fraction of the adiabatic temperature rise (recall $\Delta T_{a, i}$ are proportional to the inlet concentrations):

$$
\alpha=\frac{\Delta T_{a, 2}}{\Delta T_{a, 1}+\Delta T_{a, 2}}
$$

The critical (threshold) value separating domains of locked/separated fronts $\alpha^{s b}=\alpha\left(T_{m, i}, T_{i n}\right)$ is defined as following:

$$
\alpha^{s b}=\left(1+\frac{T_{m, 1}-T_{i n}}{T_{m, 2}-T_{m, 1}}\right)^{-1}
$$

where $T_{m, i}$ are the maximal temperature behind the $i$-th front.

A schematic diagram for an (infinitely) long reactor is shown in Figure 16 a in the plane $\Delta T_{a, 2} \mathrm{vs} \mathrm{LeV}_{f, 2}$, (recall, we are interested in the conversion of the second component). With $\alpha>\alpha^{s b}$ both fronts propagate with the same velocities depending on $\alpha$ and form a locked front (LF, Fig. 16b); with $\alpha<\alpha^{s b}$ the high temperature front propagates faster yielding the front separation (SF, Fig. 16c). Note, in a long system the SF's can be sustained if $\Delta T_{a, 1}$ exceeds the appropriate minimal value and are not restricted neither by the system length, nor by the time required for the separation. 


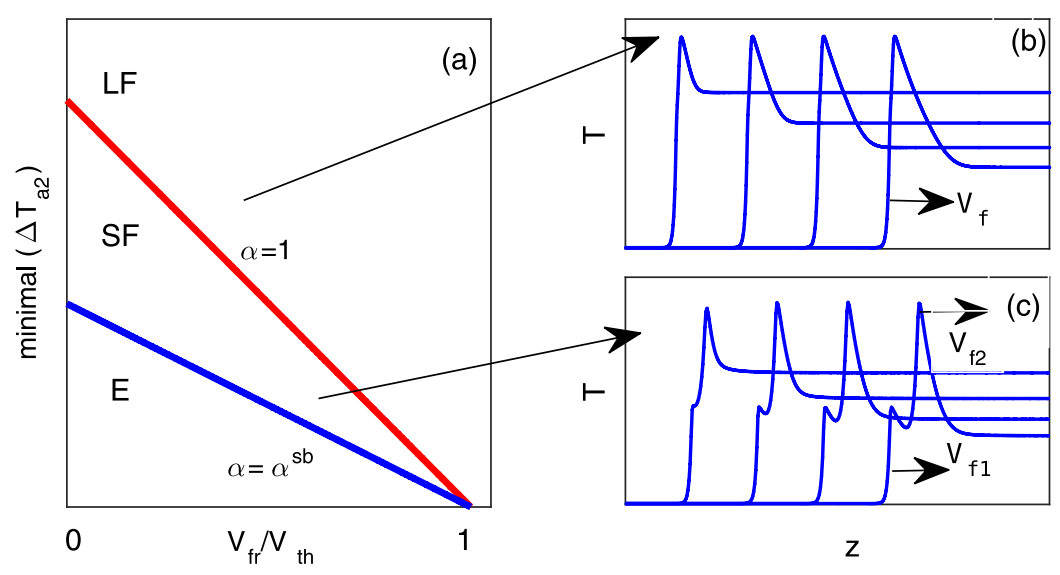

FiguRE 16. Schematic diagram showing the subdomains of sustained patterns in the plane of $\left(\Delta T_{a, 2}\right.$, vs. $V_{f, 2}$, a) and the two types dynamics of the locked propagating fronts $(\mathrm{LF}, \mathrm{b})$ and the separated fronts $(\mathrm{SF}, \mathrm{c})$. In plate (a) E denotes an extinguished state.
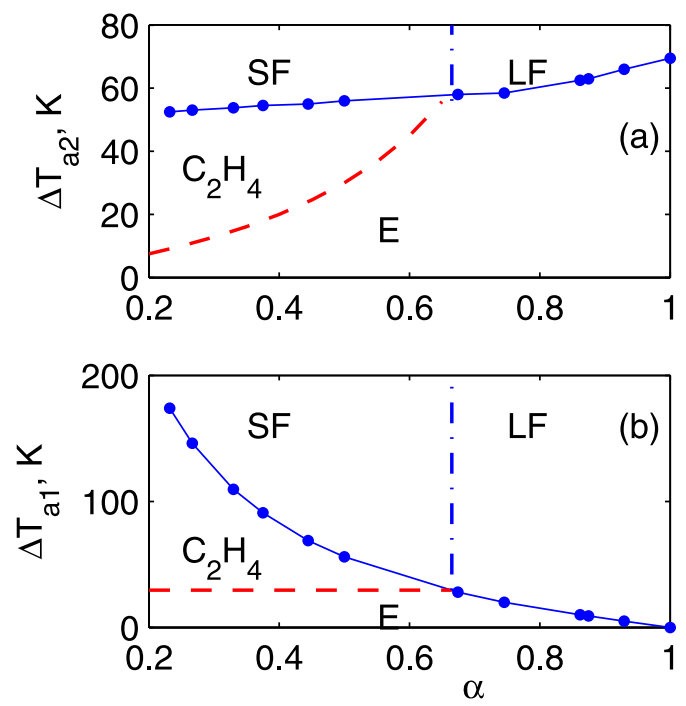

FiguRE 17. Operating domains of a LR for methane-ethylene combustion in the plane of $\Delta T_{a, 2}$, (a) and $\Delta T_{a, 1}$, (b) vs. the mixture composition defined by the parameter $\alpha=\Delta T_{a, 2} / \Delta T_{a, t o t}$. Four subdomain are shown: Locked (LF) or separated fronts (SF), total (E) or partial extinction $\left(\mathrm{C}_{2} \mathrm{H}_{4}\right.$ combustion only). Symbols connected by solid lines denote numerical simulations, dashed lines mark the extinction boundary $\left(\Delta T_{a, 1}=29.5 \mathrm{~K}\right)$, dashed-dotted lines mark the boundary between the locked and the separated front solutions. $V_{s w} / V_{t h}=0.95$. After reference [29].

We are interested now in portraying the domain that will sustain operation in a LR. The analysis of (oncethrough) long reactors, suggests that the locked front solution is preferential as both components contribute the combustion energy, while the front separation leads to an inefficient result since either the less reactive combustion extinguishes or the required total ATR is larger than in locked front solutions. The simulations were conducted for a nonadiabatic loop reactor composed of three units. Conditions leading to locked fronts in a once-through reactor (4.2) are similar to those in a LR. However, conditions that lead to the front separation may not have the same effect in a LR, as the fronts are born simultaneously after every switching and may not 
have enough time to separate before the next switching or to be washed out of the system. Recall, the switching velocity in the LR model $\left(V_{s w}\right)$ is the forcing parameter. It affects the maximal front temperatures and the separation boundary $\alpha^{s b}(4.2)$ as well (recall, $V_{s w}$ can be approximately varied between the front velocity $V_{f}$ in a long once through system and the thermal front velocity $V_{t h}$, equation (2.12)). The domain around $V_{s w} / V_{t h}=1$ is the domain with the highest potential for heat recuperation as was shown in numerous studies (e.g. [1, 37, 45]).

Typical bifurcation diagrams showing four subdomains of sustained patterns in the plane of $\Delta T_{a, 2}$, (a) and $\Delta T_{a, 1}$, (b) vs. the mixture composition defined by the parameter $\alpha$ are presented in Figure 17 for methaneethylene combustion. Plots of the individual ATR $\left(\Delta T_{a, i}\right)$ shows subdomains in which either the two reactions takes place ( $\mathrm{LF}, \mathrm{SF})$, or where only the fast reaction is sustained $\left(\mathrm{C}_{2} \mathrm{H}_{4}\right.$ combustion), or where both reactions are extinguished (E). With $V_{s w} / V_{t h} \sim 1$ the co-combustion is achieved if $\Delta T_{a, 2}$ varies between the boundaries of a pure combustion $\left(\Delta T_{a, 2}=70 \mathrm{~K}, \alpha=1\right)$ and that of a separated front pattern $\left(\Delta T_{a, 2}=54 \mathrm{~K}, \alpha=0.2\right)$. The relatively small range of $\Delta T_{a, 2}$ obtained in simulations agrees with the proposed approximation showing that the effect of the feed (cold end) temperature is marginal. Below the LF or SF boundary the slower reaction is extinguished and the system behavior is defined by the ethylene concentration, like in a single component problem: If $\Delta T_{a, 1}$ falls below the limiting value for ethylene conversion $\left(\Delta T_{a, 1}=30 \mathrm{~K}\right.$ with $\left.V_{s w} / V_{t h} \sim 1\right)$ then even the fast reaction extinguishes. Recall, the limiting $\Delta T_{a, 1}$ also defines the boundary between the locked and the separated front solutions (shown in Fig. 17 by dashed-dotted lines).

The domains presented in Figure 17 provide an insight into the map transformation upon varying of the parameters $\alpha$. Actually the 'phase' diagram is more complicated and includes also subdomains of unsteady patterns with incomplete conversion of a slow reaction superimposed on a full conversion of the fast reaction. The details of such transformation were not addressed in the literature.

\section{CONCLUDing REMARKS}

To the best of our knowledge LR is the only experimentally-tested concept of a spatio-temporally forced reactor. This concept, in the form of a pushed pulse, was tested experimentally, without control, in a catalytic surface reaction $[42,43]$. This class of diffusion-reaction problems is rarely addressed in the literature.

The loop reactor offers a significant advantage for pollutants abatement by combustion, since it can achieve a significantly higher temperatures then the once through operation. Since the rate depends exponentially on the temperature, it has the potential of abatement of highly diluted streams of HC's by oxidation to inert products. The dynamics of such a reactor, however, can be quite complex, and its operation can be sustained in a narrow domain of parameters; moreover, the kinetics, physical and transport parameters are known with some uncertainty, which requires efficient control for its operation. Various modes of control were suggested; some derived by intuition while other by a systematic approach.

This review focused on the application of combustion of diluted streams of hydrocarbons, but several other applications were considered numerically or experimentally like reversible exothermic reaction [35, 40], which require a declining temperature as reaction progresses, selective reduction of NO [12] and the production of syngas through the coupling of the exothermic partial oxidation and the endothermic steam reforming.

The technological challenges associated by the LR design, which were not addressed here, and the need for effective control make its application on a commercial scale a hard sell. Its superiority of the once-through operation is obvious and several studies demonstrated that it can achieve a higher temperatures than even the reverse flow reactor $[1,7,36,42]$, but the latter requires only two feed ports and a simple control. Designing a better control system for the LR is still a challenge, especially in domains outside that of frozen rotating waves. Initial applications of control were intuitive; later studies (see Sect. 3) attempted to carry analytical study, but that is quite a challenge in the absence of a homogeneous solution and with staionary sensors. For most of this review we considered a simple pulse solution but pointed out the existence of multiperiodic and quasiperiodic solutions. Control of a rotating train patterns were suggested by only few studies (e.g., Ref. [2, 3]).

\section{Abbreviation}

ATR - adiabatic temperature rise 
BC - boundary condition

CFR - cross-flow reactor

E - extinguished state

FP - frozen patterns (defined for an asymptotic model)

$\mathrm{HC}$ - hydrocarbons

LF - locked fronts

LR - loop reactor

NFP - non-frozen patterns (defined for a finite unit system)

NTW - network

QFP - quasi-frozen patterns

RFR - reverse flow reactor

QNFP - quasi-nonfrozen pattern

$\mathrm{SF}$ - separated fronts

VOC - volatile organic compounds

\section{REFERENCES}

[1] P. Altimari, P.L. Maffettone, S. Crescitelli, L. Russo and E. Mansusi, Nonlinear dynamics of a VOC combustion loop reactor. AIChE J. 52 (2006) 2812-2822.

[2] P. Altimari and E. Mancusi, Control of temperature wave trains in periodically forced networks of catalytic reactors for methanol synthesis. Chem. Eng. Proc.: Process Intensif. 63 (2013) 25-36.

[3] P. Altimari and E. Mancusi, Control of rotating wave trains in a looped reactor. Ind. Eng. Chem. Res. 52 (2013) $12134-12145$.

[4] P. Altimari, E. Mancusi, L. Russo and S. Crescitelli, Temperature wave-trains of periodically forced networks of catalytic reactors. AIChE J. 58 (2012) 899-913.

[5] P. Altimari, E. Mansusi and S. Crescitelli, Formation of thermal wave train in loop reactors: Stability limits and spatiotemporal structure for reversible reactions. Ind. Eng. Chem. Res. 51 (2012) 9609-9619.

[6] A.A. Barresi, M. Vanni, M. Brinkmann and G. Baldi, Control of autothermal networks of nonstationary catalytic reactors. AIChE J. 45 (1999) 1597-1602.

[7] M. Brinkmann, A.A. Barresi, M. Vanni and G. Baldi, Unsteady state treatment of very lean waste gases in a network of catalytic burners. Cat. Today 47 (1999) 263277.

[8] C.B. Broughton and C.G. Gerhold, Continuous sorption process employing fixed bed of sorbent and moving inlets and outlets, US Patent 2985589 (1961).

[9] A. Burghardt, M. Berezowski and E.W. Jacobsen, Approximate characteristics of a moving temperature front in a fixed-bed catalytic reactor. Chem. Eng. Proc. 38 (1999) 19-34.

[10] D. Fissore, Robust control in presence of parametric uncertainties: observer-based feedback controller design. Chem. Eng. Sci. 63 (2008) 1890-1900.

[11] D. Fissore and A.A. Barresi, Comparison between the reverse-flow reactor and a network of reactors for the oxidation of lean VOC mixtures. Chem. Eng. Tech. 25 (2002) 421-426.

[12] D. Fissore, A.A. Barresi and C.C. Botar-Jid, $\mathrm{NO}_{x}$ removal in forced unsteady-state chromatographic reactors. Chem. Eng. Sci. 61 (2006) 3409-3414.

[13] D. Fissore, R. Pisano and A.A. Barresi, Observer design for the selective catalytic reduction of $\mathrm{NO}_{x}$ in a loop reactor. Chem. Eng. J. 128 (2007) 181-189.

[14] D.A. Frank-Kamenetski, Diffusion and Heat Exchange in Chemical Kinetics. Princeton University Press, Princeton, NJ (1955).

[15] E.J. Gatica, J. Puzhinski and V. Hlavacek, Reaction front propagation in nonadiabatic exothermic reaction flow systems. AIChE J. 33 (1997) 819-833.

[16] T.N. Haynes and H.S. Caram, The simulated moving bed chemical reactor. Chem. Eng. Sci. 49 (1994) 5465-5472.

[17] M.A.G. Hevia, D. Fissore, S. Ordóñez, F.V. Díez and A.A.Barresi, Combustion of medium concentration CH4-air mixtures in non-stationary reactors. Chem. Eng. J. 131 (2007) 343-349.

[18] O.V. Kiselev, Theoretical Study of the Phenomena of Heat Waves Movement in Catalytic Bed. (in Russian). Russian Academy of Sciences, Institute of Catalysis (1993).

[19] G. Kolios, J. Frauhammer and G. Eigenberger, Autothermal fixed-bed reactors concepts. Chem. Eng. Sci. 55 (2000) $5945-5967$.

[20] G. Lauschke and E.D. Gilles, Circulation reaction zones in a packed-bed loop reactors. Chem. Eng. Sci. 49 (1994) 5359-5375.

[21] A.Y. Madai, O. Nekhamkina and M. Sheintuch, What is the leanest stream to sustain a nonadiabatic loop reactor: analysis and methane combustion experiments. AIChE J. 63 (2017) 2030-2042.

[22] A.Y. Madai and M. Sheintuch, Demonstration of loop reactor operation. AIChE J. 54 (2008) 2413-2422.

[23] A.Y. Madai and M. Sheintuch, Optimal design and control of nonadiabatic loop reactors. Chem. Eng. Sci. 65 (2010) 107-113.

[24] E. Mancusi, L. Russo, P. Altimari, P.L. Maffettone and S. Crescitelli, Effect of the switch strategy on the stability of reactor networks. Ind. Eng. Chem. Res. 46 (2007) 6510-6521. 
[25] E. Mancusi, P. Altimari, P. L. Maffettone, S. Crescitelli and L. Russo, Temperature and conversion patterns in a network of catalytic reactors for methanol synthesis with different switch strategies. CES 65 (2010) 4579-4590.

[26] P. Marin, D. Fissore, A.A. Barresi and S. Ordonez, Simulation of an industrial-scale process for the SCR of NOx based on the loop reactor concept. Chem. Eng. Proc. 48 (2009) 311-320.

[27] Y.S. Matros, Catalytic Process Under Unsteady-State Conditions. Elsevier, Amsterdam (1989).

[28] Y.S. Matros, G.A. Bunimovich, V.O. Strots and E.A. Mirosh, Reversed flow converter for emission control after automotive engines. Chem. Eng. Sci. 54 (1999) 2889-2898.

[29] O. Nekhamkina, A.Y. Madai, and M. Sheintuch, Front separation and 'locking' during hydrocarbons co-combustion in a loop reactor. Chem. Eng. J. 323 (2017) 618-632.

[30] O.A. Nekhamkina, A.A. Nepomnyashchy, B.Y. Rubinstein and M. Sheintuch, Nonlinear analysis of stationary patterns in convection-reaction-diffusion systems. Phys. Rev. E 61 (2000) 2436-2444.

[31] O. Nekhamkina and M. Sheintuch, Structure of operation domains of loop reactors. AIChE J. 54 (2008) 1292-1302.

[32] O. Nekhamkina and M. Sheintuch, Approximate design of loop reactors. Chem. Eng. Sci. 63 (2008) $4924-4934$.

[33] O. Nekhamkina and M. Sheintuch, Cross-flow reactor design for Fischer Tropsch synthesis. Chem. Eng. J. 372 (2019) $277-293$.

[34] L. Russo, P. Altimari, E. Mancusi, P.L. Maffettone and S. Crescitelli, Complex dynamics and spatiotemporal patterns in a network of three distributed chemical reactors with periodical feed switching. Chaos Solit. Fract. 3 (2006) 682706.

[35] R. Sheinman and M. Sheintuch, Loop reactor design and control for reversible exothermic reactions. Ind. Eng. Chem. Res. 48 (2009) 5185-5192.

[36] M. Sheintuch and O. Nekhamkina, Comparison of flow-reversal, internal-recirculation and loop reactors. Chem. Eng. J. 59 (2004) 4065-4072.

[37] M. Sheintuch and O. Nekhamkina, The asymptotes of loop reactors. AIChE J. 51 (2005) 224-234.

[38] Y. Smagina and M. Sheintuch, Control of rotating pulses in a loop reactor. J. Process Control 19 (2009) $954-963$.

[39] Y. Smagina and M. Sheintuch, Control of traveling solutions in a loop-reactor. Math. Model. Nat. Phenom. 6 (2011) 209-225.

[40] S.A. Velardi and A.A. Barresi, Methanol synthesis in a forced .unsteady state reactor network. Chem. Eng. Sci. 57 (2002) 2995-3004.

[41] A.I. Volpert, V.I. Volpert and V.I. Volpert, Traveling wave solutions of parabolic systems. AMS (1994).

[42] J. Wolff, A.G. Papathanasiou, I.G. Kevrekidis, H.H. Rotermund and G. Ertl, Spatiotemporal addressing of surface activity. Science 294 (2001) 134-137.

[43] J. Wolff, A.G. Papathanasiou, H.H. Rotermund, G. Ertl, X. Li and I.G. Kevrekidis, Gentle dragging of reaction waves. Phys. Rev. Lett. 90 (2003) 018302.

[44] V.Z. Yakhnin, A.B. Rovinsky and M. Menzinger, Differential flow instability of the exothermic standard reaction in a tubular cross-flow reactor. Chem. Eng. Sci. 49 (1994) 3257-3262.

[45] V.M. Zahn, M. Mangold, M. Krasnyk and A. Seidel-Morgenstern, Theoretical analysis of heat integration in a periodically operated cascade of catalytic fixed-bed reactors. Chem. Eng. Tech. 32 (2009) 1326-1338.

[46] V.M. Zahn, M. Mangold and A. Seidel-Morgenstern, Autothermal operation of an adiabatic simulated counter current reactor. Chem. Eng. Sci. 65 (2010) 458-465.

[47] V.M. Zahn, C.U. Yi and A.S. Morgenstern, Analysis and demonstration of a control concept for a heat integrated simulated moving bed reactor. Chem. Eng. Sci. 66 (2011) 4901-4912.

[48] Y.B. Zeldovich, G.I. Barenblatt, V.B. Librovich and G.M. Makhviladze, Mathematical theory of combustion and explosions. US Springer (1985). 